\title{
Effects of self-generated noise on estimates of detection threshold in quiet for school-age children and adults
}

\author{
Emily Buss ${ }^{1}$, Heather L. Porter ${ }^{2}$, Lori J. Leibold ${ }^{3}$, John H. Grose ${ }^{1}$, and Joseph W. Hall III ${ }^{1}$ \\ 1Department of Otolaryngology/Head and Neck Surgery, University of North Carolina at Chapel \\ Hill, Chapel Hill, NC 27599 \\ 2Department of Otolaryngology, Keck School of Medicine at USC, Los Angeles, CA 90033 \\ ${ }^{3}$ Center for Hearing Research, Boys Town National Research Hospital, Omaha, NE 68105
}

\begin{abstract}
Objectives-Detection thresholds in quiet become adult-like earlier in childhood for high than low frequencies. When adults listen for sounds near threshold, they tend to engage in behaviors that reduce physiologic noise (e.g., quiet breathing), which is predominantly low frequency. Children may not suppress self-generated noise to the same extent as adults, such that lowfrequency self-generated noise elevates thresholds in the associated frequency regions. This possibility was evaluated by measuring noise levels in the ear canal simultaneous with adaptive threshold estimation.
\end{abstract}

Design-Listeners were normal-hearing children (4.3-16.0 yrs) and adults. Detection thresholds were measured adaptively for $250-, 1000-$ and $4000-\mathrm{Hz}$ pure tones using a three-alternative forcedchoice procedure. Recordings of noise in the ear canal were made while the listeners performed this task, with the earphone and microphone routed through a single foam insert. Levels of selfgenerated noise were computed in octave-wide bands. Age effects were evaluated for four groups: 4- to 6-year-olds, 7- to 10-year-olds, 11- to 16-year-olds, and adults.

Results-Consistent with previous data, the effect of child age on thresholds was robust at 250 $\mathrm{Hz}$ and fell off at higher frequencies; thresholds of even the youngest listeners were similar to adults' at $4000 \mathrm{~Hz}$. Self-generated noise had a similar low-pass spectral shape for all age groups, although the magnitude of self-generated noise was higher in younger listeners. If self-generated noise impairs detection, then noise levels should be higher for trials associated with the wrong answer than the right answer. This association was observed for all listener groups at the $250-\mathrm{Hz}$ signal frequency. For adults and older children, this association was limited to the noise band centered on the $250-\mathrm{Hz}$ signal. For the two younger groups of children, this association was strongest at the signal frequency, but extended to bands spectrally remote from the $250-\mathrm{Hz}$ signal. For the $1000-\mathrm{Hz}$ signal frequency, there was a broadly tuned association between noise and response only for the two younger groups of children. For the 4000-Hz signal frequency, only the

Address correspondence to: Emily Buss, Department of Otolaryngology/Head and Neck Surgery, 170 Manning Dr., CB\#7070, Chapel Hill, NC 27599, USA, ebuss@ @med.unc.edu, fax: (984) 974-3499, phone: (984) 974-6484.

A preliminary report of these results was presented at The $167^{\text {th }}$ Meeting of the Acoustical Society of America. 
youngest group of children demonstrated an association between responses and noise levels, and this association was particularly pronounced for bands below the signal frequency.

Conclusions-These results provide evidence that self-generated noise plays a role in the prolonged development of low-frequency detection thresholds in quiet. Some aspects of the results are consistent with the possibility that self-generated noise elevates thresholds via energetic masking, particularly at $250 \mathrm{~Hz}$. The association between behavioral responses and noise spectrally remote from the signal frequency is also consistent with the idea that self-generated noise may also reflect contributions of more central factors (e.g., inattention to the task). Evaluation of self-generated noise could improve diagnosis of minimal or mild hearing loss.

\section{INTRODUCTION}

Performance in some auditory tasks continues to mature into adolescence (Fior 1972; Maxon and Hochberg 1982), despite the fact that cochlear function appears to be grossly mature at full-term birth (Pujol et al. 1991). This has been demonstrated for complex auditory tasks, such as detection of a tone in a masker composed of random-frequency tones (Hall et al. 2005; Oh et al. 2001) or speech recognition in the presence of a competing speech background (Bonino et al. 2013; Wightman et al. 2010), but age effects are also observed for very simple tasks. For example, low-frequency tone detection in quiet continues to mature well into childhood (Trehub et al. 1988). The time course of auditory development is thought to reflect a combination of anatomical, physiological, and cognitive factors (Abdala and Folsom 1995; Moore and Linthicum 2007; Okabe et al. 1988). The relative contributions of these factors to performance on specific psychoacoustic tasks are poorly understood, however.

A long-standing mystery in the developmental hearing literature is the finding that thresholds for detection in quiet become adult-like at younger ages for high-frequency stimuli than low-frequency stimuli (Buren et al. 1992; Elliott and Katz 1980; Müller et al. 2012; Schneider et al. 1986; Trehub et al. 1988). In one demonstration of this effect, Trehub et al. (1988) compared thresholds across a wide range of listener ages, from infancy to adulthood. The stimuli were bands of noise, with center frequencies ranging from 0.4 to 20 $\mathrm{kHz}$. At the lowest frequencies $(0.4$ and $1 \mathrm{kHz})$, children did not attain adult-like sensitivity until 10 years of age. As frequency increased, however, mature performance was achieved at younger ages. At $10 \mathrm{kHz} 5$-year-olds performed like adults, and at $20 \mathrm{kHz} 6$ - to 8-year-olds were more sensitive than adults. In a parallel study of masked detection thresholds, Schneider et al. (1989) found that children's thresholds were uniformly elevated relative to adults', with no evidence of a frequency effect. That is, frequency-dependent development seems to be restricted to thresholds in quiet. While elevated thresholds in children are often interpreted as reflecting immaturities related to selective attention, it seems unlikely that this ability would be frequency dependent or that it would vary depending on the presence of a masker. Another factor commonly cited as contributing to the prolonged development of low-frequency detection thresholds is frequency-specific maturation of middle ear compliance (Okabe et al. 1988). The present study examines the possibility that selfgenerated noise could play a role in children's relatively poor ability to detect low-frequency stimuli presented in quiet (Bargones et al. 1995; Nozza and Wilson 1984; Schneider et al. 
1986). This would be consistent with a frequency-specific effect of age in quiet but not in masking noise because self-generated noise would tend to be masked by a mid-level masker.

Whereas common sources of low-frequency noise in adults are associated with circulation, respiration and general muscle activity (Gavriely et al. 1981; Ren et al. 1995; Shaw 1974), additional factors, such as fidgeting or noisy breathing, may elevate noise in young listeners. While little is known about the effects of self-generated noise in children, several lines of evidence indicate that it interferes with detection of low-frequency stimuli in adults. Watson et al. (1972) measured pure-tone thresholds at octave frequencies between 125 and $8000 \mathrm{~Hz}$ in trained adults. They argued that masking associated with low-frequency physiologic noise in the ear canal could account for thresholds at the lowest frequency, but that additional factors were necessary to account for thresholds in the middle and high end of the spectrum. Further, Watson et al. observed that psychometric functions became steeper as signal frequency increased from 125 to $500 \mathrm{~Hz}$, but plateaued with additional increases in frequency. Shallow slopes were interpreted as reflecting contributions of noise in the ear canal at low frequencies. Support for this interpretation was provided by Moulin (1972). That study measured psychometric functions for pure-tone detection in two groups of listeners: those with normal hearing, and those with otosclerosis but normal cochlear function. Psychometric function slopes were shallower in the listeners with normal hearing than those with otosclerosis, particularly at the lowest test frequency of $80 \mathrm{~Hz}$. This result was explained in terms of low-frequency physiologic noise in normal-hearing listeners introducing variability and uncertainty into the detection task, thereby reducing the psychometric function slope. Conductive hearing loss was argued to protect the listeners with otosclerosis from these effects.

While self-generated noise is thought to impact adults' low-frequency thresholds in quiet, there is some evidence that adults engage in behaviors to reduce these effects when tasked with detecting a soft sound. These behaviors include remaining still, relaxing the lower facial muscles, delaying inspiration, and slowing the heart rate (reviewed by Stekelenburg and van Boxtel 2001). For example, Stekelenburg and van Boxtel (2001) demonstrated a correlation between low-frequency tone detection and electromyographic activity in the masticatory and lower facial muscles. Activity in these muscles could affect thresholds by introducing acoustic noise or by impairing middle ear transmission. It has also been suggested that selective attention may reduce broadband noise associated with cochlear function via activation of the efferent system (Walsh et al. 2014); in contrast to other sources of self-generated noise, cochlear noise is not predominantly low frequency.

While adults engage in behaviors that reduce their levels of self-generated noise, there is some indication that this takes practice. Loeb and Dickson (1961) reported that providing adults with practice over the course of five days improved pure-tone thresholds at $125 \mathrm{~Hz}$ by approximately $10 \mathrm{~dB}$, whereas thresholds at $3000 \mathrm{~Hz}$ remained within 1-2 dB of the baseline threshold. In contrast, learning effects were uniform across frequency when thresholds were measured in the presence of a 50-dB SPL white noise. Loeb and Dickson hypothesized that the learning effect for low-frequency tone detection in quiet reflected the development of strategies for listening in the presence of low-frequency physiologic noise. A follow-up study attempted to simulate the effects of physiologic noise at a high frequency by asking 
listeners to detect a $3000-\mathrm{Hz}$ tone masked by a narrowband noise $(3000-3200 \mathrm{~Hz})$ thought to resemble the pure-tone signal, but providing practice in this condition did not improve performance. One interpretation of these results is that practice isn't associated with learning strategies for listening in the presence of physiologic noise so much as learning strategies for suppressing that noise. If adults require practice to hone a strategy for low-frequency detection, it is reasonable to speculate that young children could require even more practice, or that their implementation of such a strategy would be less skilled than adults'. It is also possible that young children would not recognize the advantage of limiting self-generated noise.

The present experiment tested the hypothesis that young children produce more lowfrequency noise than adults when listening for soft sounds presented in quiet, and that this self-generated noise elevates thresholds and plays a role in the more protracted development at low than high signal frequencies. Pure-tone detection thresholds were measured in normal-hearing children and adults at 250,1000 and $4000 \mathrm{~Hz}$. Recordings of the noise present in the ear canal were taken during threshold estimation, and listener responses were analyzed relative to the noise levels present during the signal interval. If self-generated noise is responsible for the frequency-by-age interaction in detection thresholds, then the association between noise levels in the ear canal and the probability of detecting a signal should be strongest for the youngest listeners and the lowest signal frequency $(250 \mathrm{~Hz})$.

\section{METHODS}

\section{A. Listeners}

All listeners passed a hearing screening, with thresholds of $20 \mathrm{~dB}$ HL or better for octave frequencies between 250 and $8000 \mathrm{~Hz}$ (ANSI 2010). Tympanometry was not performed. A total of 37 children was tested, with ages ranging from 4.3 to $16.0 \mathrm{yrs}$ (mean $8.9 \mathrm{yrs}$ ). In addition, one 5-year-old began testing but chose to withdraw from the study before completing data collection. In some cases data are described for three subgroups of children: 4- to 6-year-olds ( $n=15), 7$ - to 10-year-olds $(n=12)$, and 11- to 16-year-olds $(n=10)$. These groupings are approximately equally spaced on the log transform of age in years. In addition to child listeners, a group of 10 adults was tested, with ages ranging from 21.5 to $60.3 \mathrm{yrs}$ (mean 36.6 yrs).

\section{B. Stimuli}

The signal was a 200-ms tone, gated using 10-ms raised-cosine ramps, and its frequency was 250,1000 , or $4000 \mathrm{~Hz}$. The detection task was a three-alternative forced-choice. Each interval lasted $1 \mathrm{sec}$, and the signal was temporally centered in one of the three intervals, selected at random. A video display showed three frogs that opened their mouths in sequence, left to right, to indicate each of the three intervals. The listener indicated which interval contained the tone by selecting the associated picture using a touchscreen. After the listener's response, the frog associated with the correct response performed an animation in which the frog's tongue reached up and caught a fly. 
Concurrent with psychophysical threshold estimation, noise levels in the ear canal were measured. Recordings began $1 \mathrm{sec}$ prior to the beginning of the first interval, spanned the three 1-sec listening intervals, and ended $1 \mathrm{sec}$ after completion of the final interval. The 5sec recording associated with each trial was saved to disk for subsequent analysis, allowing interval-by-interval assessment of the association between a listener's ability to detect the signal and the level of self-generated noise. In addition, a 5-sec Gaussian noise was presented at $67 \mathrm{~dB}$ SPL at the beginning and end of each threshold estimation track. Recordings of this calibration stimulus were examined to evaluate stability of the foam insert over the course of the track.

\section{Hardware}

The signal tone was played out of a real-time processor (RP2, TDT), routed to a headphone buffer (HB7, TDT), and presented via an insert earphone (ER-10C, Etymotic). Recordings of sound levels in the ear canal were made using a microphone embedded in the same assembly as the earphone. These recordings were amplified (ER-10C, Etymotic), routed into the real-time processor (RP2, TDT), and sampled at a rate of $12207 \mathrm{~Hz}$. Presentation levels for the tonal signals were calibrated using a 2cc coupler and a sound level meter (800B, Larson Davis). The sensitivity of the recording microphone was calibrated using octavewide bands of noise with center frequencies of 62 to $4000 \mathrm{~Hz}$. Estimates of the noise floor of the recording setup were made in a $2 \mathrm{cc}$ coupler.

\section{Procedures}

The foam insert was placed in the ear canal by the experimenter, with an intermediate insertion depth (Bell-Lehmkuhler et al. 2009). The cable associated with the earphone/ microphone assembly was clipped to the back of the listener's collar. If the foam insert became dislodged during a threshold run, it was replaced and data collection continued. This only occurred with child listeners. While replacing the insert could change its position in the ear canal, and therefore the levels of sound delivered and recorded, it was reasoned that such a change would be evident in the calibration results. One factor that may have affected the stability of the insert is the weight of the earphone/microphone assembly 1 .

Thresholds were determined using a two-down, one-up adaptive track that estimates the signal level associated with $71 \%$ correct. The initial step size was $8 \mathrm{~dB}$, reduced to $4 \mathrm{~dB}$ after the first track reversal, and further reduced to $2 \mathrm{~dB}$ after the second track reversal. A track continued until eight reversals had been obtained, and the mean of the last six reversals served as the estimate of threshold. Each listener completed between one and four threshold estimation tracks for each of the three signal frequencies, depending on variability of threshold estimates, scheduling limitations, and the listener's ability to stay on task. These tracks were quasi-randomly interleaved, with the caveat that listeners completed one track at each signal frequency before proceeding to the second estimate, and completed a second estimate at each frequency before proceeding to the third estimate. Each track lasted approximately 5 minutes.

\footnotetext{
${ }^{1}$ The earphone/probe assembly is housed in a thick plastic case which is approximately $1 "$ in diameter, making it bulkier and slightly heavier than a standard insert earphone.
} 
All testing was conducted by the second author, an experienced pediatric audiologist. Age appropriate instructions were provided, specifying that the listener should sit quietly and listen carefully. The experimenter was present in the booth during testing to reinstruct the listeners as needed. Prior to data collection, child listeners were provided with practice on the detection task. In these trials the signal was a 50-dB-SPL tone at $1000 \mathrm{~Hz}$. Practice continued until the child could reliably indicate the signal interval at least three times in a row. All listeners reached this level of performance in fewer than ten practice trials. Once data collection began, children were given a brief break after every two or three threshold estimation tracks, and adults completed all tracks in one sitting. All procedures were approved by the Institutional Review Board at the University of North Carolina at Chapel Hill, and listeners were compensated at a rate of $\$ 15$ per hour.

\section{Data Analysis}

Each recording of self-generated noise was filtered into six octave-wide bands, with center frequencies of 125 to $4000 \mathrm{~Hz}$. This was achieved using FIR filters with 5-Hz resolution. The noise level in each band was evaluated, including but not limited to the band centered on signal frequency, in order to evaluate the frequency specificity of the relationship between noise and pure-tone sensitivity. If noise affects sensitivity through energetic masking (Fletcher 1940), then the effect should be tuned to the frequency region of the signal. One caveat to this prediction is that many self-generated noises (e.g., breathing) are broadband, reducing the ability to observe frequency tuning. In contrast, the relationship between noise and pure-tone sensitivity could reflect a third factor, such as restless inattention, in which case the effect would not be tuned in frequency. Statistical tests used a criterion of $a=0.05$, and significance was evaluated two-tailed. Greenhouse-Geisser corrections were applied in repeated-measures analysis of variance (rmANOVA), as indicated. The log of listener age was used to evaluate age effects, based on the observation that development is expected to be more rapid in younger listeners (Mayer and Dobson 1982; Moller and Rollins 2002).

\section{RESULTS}

Younger listeners were not able to complete as many threshold estimation tracks as older listeners. On average, 4- to 6-year-olds completed 2.5 runs per condition, 7- to -10-year-olds completed 3.0 runs per condition, 11- to 16-year-olds completed 3.1 runs per condition, and adults completed 3.3 runs per condition. All data collected for each listener were included in the analyses reported below unless specified otherwise. Repeating the analyses excluding all but the first adaptive track for each listener at each frequency reduced the number of significant results, but did not change the general findings.

Figure 1 shows the mean detection thresholds for individual children plotted as a function of age in years on a log scale. Filled symbols at the far right indicate mean adult thresholds, with error bars showing one standard deviation around the mean. Each panel shows results for a different signal frequency. Lines are fits to the children's thresholds as a function of log age, indicating marked improvement with increasing age at $250 \mathrm{~Hz}\left(\mathrm{r}^{2}=0.66, \mathrm{t}=-8.24, \mathrm{p}<\right.$ $0.001)$, more modest improvement at $1000 \mathrm{~Hz}\left(\mathrm{r}^{2}=0.33, \mathrm{t}=-4.12, \mathrm{p}<0.001\right)$, and a nonsignificant trend for improvement with age at $4000 \mathrm{~Hz}\left(\mathrm{r}^{2}=0.08, \mathrm{t}=-1.70, \mathrm{p}=0.098\right)$. A 
repeated-measures general linear model was fitted to the child data, with three levels of frequency and log of age as a continuous variable. There was a main effect of frequency $\left(\mathrm{F}_{2,70}=56.05, \mathrm{p}<0.001, \eta_{\mathrm{p}}^{2}=0.62\right)$ and age $\left(\mathrm{F}_{1,35}=33.86, \mathrm{p}<0.001, \eta_{\mathrm{p}}^{2}=0.49\right)$, as well as an interaction between frequency and age $\left(\mathrm{F}_{2,70}=16.45, \mathrm{p}<0.001, \eta_{\mathrm{p}}^{2}=0.32\right)$. This interaction reflects the larger effects of age at the lower frequencies.

Children's thresholds were compared to adults' with rmANOVA, with four levels of age group (4-6 yrs, 7-10 yrs, 11-16 yrs, and adults) and three levels of signal frequency (250, 1000 , and $4000 \mathrm{~Hz})$. This analysis resulted in a main effect of frequency $\left(\mathrm{F}_{2,86}=348.66, \mathrm{p}<\right.$ $\left.0.001, \eta_{\mathrm{p}}^{2}=0.89\right)$, a main effect of age group $\left(\mathrm{F}_{3,43}=11.53, \mathrm{p}<0.001, \eta_{\mathrm{p}}^{2}=0.45\right)$, and a significant interaction between frequency and age group $\left(\mathrm{F}_{6,86}=7.35, \mathrm{p}<0.001, \eta_{\mathrm{p}}^{2}=0.34\right)$. Simple main effects testing was performed to evaluate this interaction. Thresholds at $250 \mathrm{~Hz}$ were elevated relative to adults' for 4 - to 6-year-olds $(\mathrm{p}<0.001)$ and 7 - to 11-year-olds $(\mathrm{p}=$ 0.042 ), but not 11 - to 16 -year-olds ( $\mathrm{p}=0.655$ ). Thresholds at $1000 \mathrm{~Hz}$ were elevated relative to adults' for 4- to 6-year-olds ( $\mathrm{p}=0.007$ ), but not for 7- to 11-year-olds ( $\mathrm{p}=0.401$ ) or 11 - to 16-year-olds ( $\mathrm{p}=0.162$ ). Mean thresholds at $4000 \mathrm{~Hz}$ tended to be lower in children than adults; this difference was non-significant for 4- to 6-year-olds ( $\mathrm{p}=0.680)$ and 7- to 11-yearolds $(\mathrm{p}=0.394)$, but it did reach significance for 11- to 16-year-olds $(\mathrm{p}=0.033)$.

Analysis of the ear canal recordings assumes that the probe assembly is in a fixed position throughout a threshold estimation run. To evaluate this assumption the level of the calibration noise at the beginning and at the end of each run was compared. While listeners were instructed to stay quiet and still during these measurements, some of the child listeners spoke or shifted position. This was particularly common at the end of a threshold estimation track, presumably because the second calibration noise marked the end of the listeners' task. In order to evaluate the stability of the probe, it was desirable to exclude these instances of self-generated noise. To that end, calibration recordings were transformed into power, the 100-ms running average was computed, and the minimum value for each calibration interval was used to estimate the level of the calibration stimulus. This procedure was intended to minimize the effects of transient self-generated noise. The calibration level was compared for the intervals at the beginning and end of each threshold estimation track. These values were within $3 \mathrm{~dB}$ for $100 \%$ of the tracks obtained with adults and $89 \%$ of tracks obtained with children, with the majority of values greater than $3 \mathrm{~dB}$ found in data for the youngest listeners. Tracks associated with discrepancies of more than $3 \mathrm{~dB}$ between initial and final calibration levels were omitted from further analysis ${ }^{2}$.

For the tracks meeting the 3-dB stability criterion, the calibration noise was on average 2.8$\mathrm{dB}$ lower for adults than children. This observation was confirmed with rmANOVA, including four levels of age group (4-6 yrs, 7-10 yrs, 11-16 yrs, and adults) and six levels of band center frequency $(125-4000 \mathrm{~Hz})$. There was a main effect of age group $\left(\mathrm{F}_{3,43}=5.70, \mathrm{p}\right.$ $\left.=0.002, \eta_{\mathrm{p}}^{2}=0.28=0.28\right)$ and frequency $\left(\mathrm{F}_{1.8,79.0}=404.23, \mathrm{p}<0.001, \eta_{\mathrm{p}}^{2}=0.90\right)$, but no

\footnotetext{
${ }^{2}$ Threshold data were reanalyzed, omitting tracks associated with a change of $3 \mathrm{~dB}$ or more between initial and final calibration levels. Results were the same as reported for the full dataset.
} 
interaction between frequency and age group $\left(\mathrm{F}_{5.5,79.0}=1.52, \mathrm{p}=0.189, \eta_{\mathrm{p}}^{2}=0.10\right)$. Post-hoc contrasts between sequential age groups indicated that the 11- to 16-year-olds differed from adults ( $p=0.007)$, but comparisons between sequential groups of children were not significant ( $p>=0.670$ ). These data indicate that the effective presentation levels associated with the pure-tone signals were likely higher for children than adults, consistent with the better thresholds for children than adults at $4000 \mathrm{~Hz}$. The absence of an interaction means that differences in the effect of age across signal frequency cannot be attributed to differences in stimulus level in the ear canal.

One goal of the present study was to characterize the noise in the ear canal when a listener was trying to detect a signal. Listeners may not maintain high levels of vigilance once a target has been heard. Levels characteristic of attentive listening were therefore based on recordings made prior to the interval identified by the listener as containing the signal interval. In cases where the response interval occurred after the signal interval, the frequency band centered on the signal was omitted from the estimate of self-generated noise in the associated interval; that is, the band containing the signal itself was not included in the estimate of self-generated noise. Further, levels were measured after the second track reversal, when the signal level was near the listener's detection threshold. The rationale was to omit supra-threshold trials and characterize behaviors elicited by challenging trials. Mean levels per band were computed for each listener, and the means across listeners in each group for each octave-wide analysis band are shown in Figure 2. The filled area at the bottom of the panel shows the noise floor measured in the coupler. Mean values of selfgenerated noise and associated standard deviations are also reported in Table 1.

Consistent with previous data, self-generated noise measured in the present study had a lowpass spectral shape. Whereas levels tended to be higher overall in the two youngest groups of children, levels were similar for 11- to 16-year-olds and adults. Effects of age and frequency were evaluated with rmANOVA, including four levels of age group and six levels of band center frequency. There was a main effect of age group $\left(\mathrm{F}_{3,43}=27.94, \mathrm{p}<0.001\right.$, $\left.\eta_{\mathrm{p}}^{2}=0.66\right)$, a main effect of frequency $\left(\mathrm{F}_{2.5,106.4}=1196.00, \mathrm{p}<0.001, \eta_{\mathrm{p}}^{2}=0.97\right)$, and a significant interaction between age group and frequency $\left(\mathrm{F}_{7.4,106.4}=8.17, \mathrm{p}<0.001\right.$, $\eta_{\mathrm{p}}^{2}=0.36$ ). Simple main effects testing indicated that results differed between each sequential age group of children ( $\mathrm{p} \leq 0.014$ ), but that 11- to 16-year-olds did not differ from adults ( $\mathrm{p}$ $>=0.111)$; the one exception was at $4000 \mathrm{~Hz}$, where 7- to 10-year-olds differed from adults ( $p<0.001$ ), but the two older groups of children did not ( $p>=0.064)$. The group-byfrequency interaction reflects the fact that levels measured in the four groups converged at the high end of the spectrum, which may be due to the fact that self-generated noise levels approached the noise floor of the recording procedure in this spectral region.

As expected, noise recordings tended to be lower before the listener heard the signal than after. This was demonstrated by comparing noise levels before and after the interval identified by the listener as the signal interval, with noise levels computed as described above. The differences in self-generated noise before and after the response interval are reported for each age group and frequency in Table 2. In all groups, the mean change in the level of self-generated noise was larger for low than high frequencies, but this effect was 
smaller for younger listeners. An rmANOVA was performed, with four levels of age group and six levels of frequency. This resulted in a main effect of age group $\left(F_{3,43}=2.89, p=\right.$ $\left.0.046, \eta_{\mathrm{p}}^{2}=0.17\right)$, a main effect of frequency $\left(\mathrm{F}_{2.5,106.1}=102.29, \mathrm{p}<0.001, \eta_{\mathrm{p}}^{2}=0.70\right)$, and a significant interaction between age group and frequency $\left(\mathrm{F}_{7.4,106.1}=6.85, \mathrm{p}<0.001\right.$, $\eta_{\mathrm{p}}^{2}=0.32$ ). A set of four t-tests evaluated whether the change in level at the $250-\mathrm{Hz}$ frequency was significantly greater than zero; this frequency was chosen because the a priori expectation was for greater effects of self-generated noise at the lowest signal frequencies. The results were significant for all age groups ( $\mathrm{p}<=0.002$ ). These observations are consistent with the idea that both children and adults suppress self-generated noise when listening for a low-level stimulus, but that younger listeners do so to a lesser degree than adults.

The primary question of interest in the present study was whether the higher levels of lowfrequency self-generated noise play a role in the frequency-dependent threshold elevation seen in young children. Preliminary evidence for this was seen in the parallel age effects in both self-generated noise and low-frequency thresholds. Across all listeners, the correlation between self-generated noise and detection threshold was significant at $250 \mathrm{~Hz}(\mathrm{r}=0.80, \mathrm{p}<$ $0.001)$ and $1000 \mathrm{~Hz}(\mathrm{r}=0.60, \mathrm{p}<0.001)$, but failed to reach significance at $4000 \mathrm{~Hz}(\mathrm{r}=$ $0.01, p=0.925$ ). If self-generated noise plays an important role in determining thresholds, then the level of self-generated noise for individual children could be positively correlated with threshold apart from effects of listener age. This was evaluated by computing the partial correlation between noise levels in the band centered on the signal ${ }^{3}$ and thresholds, controlling for log of age. The resulting correlations were significant at $250 \mathrm{~Hz}(\mathrm{r}=0.43, \mathrm{p}=$ $0.010)$ and at $1000 \mathrm{~Hz}(\mathrm{r}=0.41, \mathrm{p}=0.013)$, but not at $4000 \mathrm{~Hz}(\mathrm{r}=-0.01, \mathrm{p}=0.951)$. These results are consistent with self-generated noise playing a causal role in behavioral thresholds at 250 and $1000 \mathrm{~Hz}$, rather than both measures reflecting overall maturation. Within adult data, the correlation between noise levels in the band centered on the signal and thresholds was significant at $250 \mathrm{~Hz}(\mathrm{r}=0.66, \mathrm{p}=0.026)$, but not at $1000 \mathrm{~Hz}(\mathrm{r}=0.10, \mathrm{p}=0.78)$ or $4000 \mathrm{~Hz}(\mathrm{r}=-0.37, \mathrm{p}=0.29)$. In contrast to data from children, this result suggests that the association between self-generated noise and thresholds appears to be limited to $250 \mathrm{~Hz}$ in adults.

If self-generated noise noise affects thresholds, then there should be an association between noise levels recorded in the ear canal near the signal frequency and the probability of the listener correctly identifying the signal interval. These values were compiled for the trials associated with a particular listener and signal frequency, omitting trials before the second reversal of each track. Recordings from the octave band centered on the signal frequency were notch-filtered, with a bandstop region $\pm 75 \mathrm{~Hz}$ around the signal ${ }^{4}$, to prevent the signal itself from overshadowing self-generated noise. While this technique eliminates noise in the immediate vicinity of the signal, most sources of noise are relatively broadband, and the present protocol does not allow for more sophisticated signal cancelation. If self-generated

\footnotetext{
${ }^{3}$ Within child data, there was a strong correlation between noise levels at 250,1000 and $4000 \mathrm{~Hz}(\mathrm{r}=0.80$ to $\mathrm{r}=0.91, \mathrm{p}<0.001)$. As a consequence, the correlation between threshold and noise level was similar regardless of the frequency region used to calculate selfgenerated noise.

${ }^{4}$ The spectrum of the gated signal was approximately $40 \mathrm{~dB}$ down at the edges of this bandstop filter.
} 
noise energetically masked the signal, then on average the noise in the octave-wide band centered on the signal should be higher when the listener's response was wrong than when it was right. If low-frequency self-generated noise is responsible for the prolonged development of low-frequency detection, then the "wrong-right" difference should be largest for analysis bands near the target frequency in the low-frequency data of the youngest listeners.

Figure 3 shows the mean difference in self-generated noise levels during signal trials for which the listener provided the wrong response compared to those for which the listener provided the right response. Symbol shape reflects the listener group, as indicated in the legend. Each panel shows results for a different signal frequency. The pattern of wrong-right level differences depended on both listener group and signal frequency. Results from the analysis band centered on the signal, highlighted with grey symbol shading in Figure 3, are considered first. The significance of these age-by-frequency effects was evaluated via rmANOVA, with four levels of age group and three levels of signal frequency. This resulted in a main effect of age group $\left(\mathrm{F}_{3,40}=8.21, \mathrm{p}<0.001, \eta_{\mathrm{p}}^{2}=0.38\right)$, a main effect of signal frequency $\left(\mathrm{F}_{2,80}=13.75, \mathrm{p}<0.001, \eta_{\mathrm{p}}^{2}=0.626\right)$, and a significant interaction between age group and frequency $\left(\mathrm{F}_{6,80}=2.78, \mathrm{p}=0.017, \eta_{\mathrm{p}}^{2}=0.17\right)$. T-tests were used to evaluate the prediction that the wrong-right difference would be positive for lower signal frequencies and younger listeners. At $250 \mathrm{~Hz}$ the wrong-right difference was greater than zero for all four listener groups ( $\mathrm{p} \leq 0.032$ ). At $1000 \mathrm{~Hz}$ the difference score was greater than zero for child groups ( $\mathrm{p} \leq 0.038$ ), but not adults $(\mathrm{p}=0.532$ ). At $4000 \mathrm{~Hz}$ the wrong-right difference was significantly greater than zero in 4 - to 6 -year olds $(\mathrm{p}=0.040)$, but not for older listeners $(\mathrm{p} \geq$ 0.116). This analysis confirms the observation that the wrong-right difference depends on frequency in different ways for younger and older listeners.

The results shown in Figure 3 also indicate an effect of signal frequency on the degree to which the wrong-right difference appeared to be tuned to the signal frequency. For the 250$\mathrm{Hz}$-signal data, there was a peak in the wrong-right difference at the signal frequency for all groups, particularly evident in the data of older listeners. Apart from this peak, the difference scores hovered around zero for both 11- to 16-year-olds and adults, whereas the difference score tended to be positive across frequency for the two younger groups. For the 4000-Hzsignal data there was no evidence of a peak in the wrong-right difference at the signal frequency. In this dataset the difference score was close to zero across all analysis bands for all groups except the 4- to 6-year-olds, for whom the difference tended to be positive at all frequencies. For the 1000-Hz-signal data, the pattern of results was intermediate between those for the $250-$ and $4000-\mathrm{Hz}$ data, in that there was relatively modest evidence of tuning to the signal frequency in the child data.

Whereas effects of self-generated noise at $250 \mathrm{~Hz}$ were anticipated at the outset of the experiment, one unexpected result was the finding of large wrong-right differences in the 1000-Hz data for 4- to 6-year olds and 7- to 10-year olds. Given the smaller effects of age on detection thresholds in this spectral region as well as the lower levels of physiologic noise at mid frequencies, a smaller wrong-right difference was expected relative to the effect size at $250 \mathrm{~Hz}$. While the present study was not designed to identify particular sources of self- 
generated noise, an informal evaluation of the recordings indicate that noise levels may have been particularly elevated in the mid-frequencies (e.g., $1000 \mathrm{~Hz}$ ) by swallowing and mouth breathing.

\section{DISCUSSION}

The present study set out to test the hypothesis that the protracted maturation of lowfrequency tone detection in quiet is due, at least in part, to higher levels of low-frequency self-generated noise in young children. The approach used to test this hypothesis was to measure thresholds while simultaneously measuring noise levels in the ear canal using an insert earphone coupled with a microphone, and then to look for an association between levels of self-generated noise and behavioral responses.

\section{A. Thresholds}

Thresholds measured in the present study are consistent with previous data showing a prolonged time-course of development for the detection of low-frequency stimuli. Within child listeners, the association between age and signal level was robust at $250 \mathrm{~Hz}$, somewhat weaker at $1000 \mathrm{~Hz}$, and non-significant at $4000 \mathrm{~Hz}$. Compared to adults, thresholds of the 4to 6-year-olds were elevated at 250 and $1000 \mathrm{~Hz}$, but not at $4000 \mathrm{~Hz}$. Thresholds of 7- to 10year-olds were elevated at $250 \mathrm{~Hz}$, but not at 1000 or $4000 \mathrm{~Hz}$. Thresholds of 11- to 16-year olds were adult-like at both 250 and $1000 \mathrm{~Hz}$, and they were better than those of adults at $4000 \mathrm{~Hz}$. While all listeners passed a screening audiogram, we cannot definitively rule out the possibility that some may have had mild cases of otitis media (OM). Several aspects of the results make it highly unlikely that a greater incidence of OM in younger listeners accounts for the age effects observed here, however. First, the effect of age on $250-\mathrm{Hz}$ thresholds is relatively consistent across individual children within an age group, inconsistent with the possibility that a subset of young children with OM could have driven the age effect. Second, while OM is sometimes associated with elevated $250-\mathrm{Hz}$ thresholds, this is not always the case; thresholds are often elevated uniformly across frequency (Fria et al. 1985; Handžić et al. 2012). The pattern of hearing loss associated with OM is therefore an unlikely explanation for the frequency-dependent effects of age observed here. Finally, the association between noise levels and behavioral responses, discussed below, is inconsistent with OM driving the effect of age; if anything, conductive hearing loss would be expected to reduce effects of self-generated noise (Moulin 1972).

Thresholds from the present study are broadly consistent with those of Trehub et al. (1988). That study concluded that children's detection of octave-wide bands of noise was not adultlike until 10 years of age for bands at 400 and $1000 \mathrm{~Hz}$, but it was adult-like by 8 years of age for bands at 2000 and $4000 \mathrm{~Hz}$. Although the dataset of Trehub et al. (1988) is arguably the most comprehensive demonstration of frequency-specific developmental effects collected under controlled laboratory conditions, comparisons between their data and those of the present experiment are undermined by procedural differences across studies, not the least of which is the use of tones in the present study. There are several datasets in the literature on pure-tone detection in quiet that can be compared to the present data, however. Elliott and Katz (1980) measured 500- and 2000-Hz pure-tone thresholds in children and 
adults and found that thresholds of typical 6-year-olds were 15.9 and $13 \mathrm{~dB}$ above those of adults, respectively. Using line fits to estimate thresholds for 6-year-olds in the present datase $^{5}$, the associated child-adult differences were $12.2 \mathrm{~dB}$ at $250 \mathrm{~Hz}, 5.2 \mathrm{~dB}$ at $1000 \mathrm{~Hz}$, and $-1.5 \mathrm{~dB}$ at $4000 \mathrm{~Hz}$. That is, it appears that the age effect was smaller and the effect of frequency was more robust in the present data than that observed by Elliott and Katz (1980).

Several clinical datasets are consistent with an effect of frequency on the maturation of detection in quiet. A number of large population-based studies indicate elevated lowfrequency pure-tone detection thresholds in young children (reviewed by Haapaniemi 1996). Many of these studies evaluated representative samples of children, irrespective of middle ear status or hearing history. However, similar effects are seen in data on otologically normal children without a history of acoustic trauma (Buren et al. 1992; Haapaniemi 1996; Müller et al. 2012). Two studies included otologically normal children approximately 7 years of age: Müller et al. (2012) reported thresholds for 6- to 8-year-olds ( $\mathrm{n}=91$ ), and Haapaniemi (1996) reported thresholds for 6- to 9-year-olds $(n=156)$. Because both studies reported their results in $\mathrm{dB} \mathrm{HL}$, the child-adult difference can be inferred relative to $0 \mathrm{~dB} \mathrm{HL}$. While the thresholds reported by Müller et al. (2012) are approximately $5 \mathrm{~dB}$ higher than those reported by Haapaniemi (1996), the slope of the child-adult difference as a function of frequency was similar. In both cases the child-adult difference dropped by about $5 \mathrm{~dB}$ between 250 and $4000 \mathrm{~Hz}$. This can be compared to a 12.6-dB reduction in the child-adult difference for 7-year-olds in the present dataset, based on line-fits to the data.

These comparisons to published data indicate that the effect of frequency on the child-adult threshold difference in the present study is large relative to that previously observed. Whereas the previous studies used circumaural or supra-aural earphones, the present study used insert phones. These transducer differences could affect both the level of the stimuli presented to the listener and the magnitude of self-generated noise present in the ear canal. While occlusion and the associated effects on physiologic noise differ across these transducers, the spectral shape of physiologic noise under each is broadly similar (Berger and Kerivan 1983). Stimulus level is affected by characteristics of the transducer, the length and volume of the ear canal, and the impedance of the tympanic membrane (reviewed by Voss and Herrmann 2005). While the real-ear-to-coupler difference for supra-aural earphones is relatively adult-like over the age range of listeners tested in the present study (Lewis and Stelmachowicz 1993), it differs with child age for insert earphones (Feigin et al. 1989). For example, Feigin et al. (1989) presented tones using insert earphones, calibrated using a $2 \mathrm{cc}$ coupler, and measured the resulting levels in the listener's ear canal using a probe microphone. At the frequencies examined in the present study, the real ear to coupler difference in adults ranged from $-5 \mathrm{~dB}(250 \mathrm{~Hz})$ to $9 \mathrm{~dB}(8000 \mathrm{~Hz})$. The mean values for 4to 5-year-olds were approximately $1-3 \mathrm{~dB}$ above those of adults, with larger differences in younger children and infants. This result is consistent with the $2.8-\mathrm{dB}$ higher calibration noise measured in children than adults. It is somewhat surprising that this difference was consistent for the three groups of children, since the older children would be expected to have more adult-like external and middle-ear anatomy. The higher effective presentation

${ }^{5}$ Elliott \& Katz (1980) did not report the mean age of their 6-year-old group, so an age of 6.5 yrs was assumed for this comparison. 
level in children would tend to reduce the child-adult difference in detection thresholds, so transducer differences across studies cannot explain the larger than expected developmental effects observed in the present dataset.

Differences in effective stimulus level could explain the unexpected finding that thresholds for the oldest children were significantly lower than those of adults at $4000 \mathrm{~Hz}$. Previous studies have shown that thresholds of children are lower than those of adults at very high frequencies (e.g., $20 \mathrm{kHz}$; Trehub et al. 1989), a finding that has been interpreted as reflecting reduced sensitivity at these frequencies in adults due to noise exposure and normal aging. This explanation does not convincingly account for the present results, however, because the trend for better performance in older children than adults is evident at all three frequencies. The threshold difference between adults and 11- to 16-year-olds was $1.2 \mathrm{~dB}$ at $250 \mathrm{~Hz}, 3.9 \mathrm{~dB}$ at $1000 \mathrm{~Hz}$, and $5.5 \mathrm{~dB}$ at $4000 \mathrm{~Hz}$. One possibility is that low thresholds in the oldest children could be due to a higher effective signal level compared to adults, as reflected in the higher levels of calibration noise across frequency. This idea receives some support from the finding that $4000-\mathrm{Hz}$ thresholds are no longer significantly different for 11to 16-year-olds and adults after applying a 2.8 - $\mathrm{dB}$ correction factor $(\mathrm{p}=0.216)$.

\section{B. Self-generated noise and its effect on thresholds}

Self-generated noise was low pass in nature for adult listeners, consistent with previous reports of physiological noise in adults (reviewed by Shaw 1974). A similar spectral shape was observed for the three groups of children, although the level of noise tended to be higher in younger listeners. For example, the difference in self-generated noise levels between adults and 4- to 6-year-olds was 15.4-17.0 dB between 125 and $1000 \mathrm{~Hz}$, falling to $12.4 \mathrm{~dB}$ at $2000 \mathrm{~Hz}$ and $6.6 \mathrm{~dB}$ at $4000 \mathrm{~Hz}$. These findings are broadly consistent with the observation that the noise floor in studies of otoacoustic emissions tends to drop with increasing age, from infancy through childhood (Lonsbury-Martin et al. 1994; Norton and Widen 1990), as well as the finding that the effect of age on the noise floor is larger at lower frequencies (Lonsbury-Martin et al. 1994).

The smallest child-adult differences were observed at the high end of the spectrum, where adults' levels of self-generated noise approached the noise floor. If the noise floor of the recording hardware causes an over estimate of high-frequency noise in adults, then it is possible that the smaller child-adult difference at high frequencies is an artifact. In any case, the child-adult differences in self-generated noise levels are robust at the lowest frequencies, the region where self-generated noise is most likely to limit sensitivity. Another factor to consider is that the coupling of the foam eartip to the ear canal can affect the amplitude of self-generated noise. In adults, the placement of the insert has a large impact on the level of physiologic noise measured in the ear canal, with effects on the order of $10 \mathrm{~dB}$ or more (Berger and Kerivan 1983); deeper insertions, into the bony portion of the ear canal, are associated with lower noise levels. While every attempt was made to place inserts at a consistent depth across listeners, it is possible that either the placement or the external ear anatomy of younger listeners could contribute to the group differences observed.

If peripheral masking associated with self-generated noise were responsible for threshold differences as a function of age, then group differences in self-generated noise in the band 
centered on the signal should be comparable in magnitude to the differences in thresholds, after applying the $2.8-\mathrm{dB}$ calibration correction factor. This expectation was met for $250-\mathrm{Hz}-$ signal data. Comparing 4- to 6-year-olds to adults, mean noise levels in the analysis band centered on $250 \mathrm{~Hz}$ differed by $17.0 \mathrm{~dB}$, and mean $250-\mathrm{Hz}$ thresholds differed by $18.0 \mathrm{~dB}$. The correspondence between group differences in self-generated noise and thresholds was not as close at the two higher signal frequencies. For 1000-Hz-signal data, mean noise levels in the analysis band centered on the signal differed by $16.3 \mathrm{~dB}$, and mean thresholds differed by $10.1 \mathrm{~dB}$. For the $4000-\mathrm{Hz}$-signal data, mean noise levels differed by $6.5 \mathrm{~dB}$, and mean thresholds were 3.0-dB higher for 4- to 6-year-olds than adults. The finding of more modest effects of age on threshold than self-generated noise at 1000 and $4000 \mathrm{~Hz}$ is likely due at least in part to reduced audibility of self-generated noise in these frequency regions; while young children generate more mid- and high-frequency noise than adults, some of that noise is below threshold, and so does not result in masking.

If self-generated noise affects thresholds via energetic masking, then one might expect higher levels of noise in the band centered on the signal for trials associated with the wrong answer than trials associated with the right answer. This hypothesis was evaluated based on trials after the second track reversal. This approach omits clearly supra-threshold signal trials, where masking is less likely, due to high signal levels, and the listener might not be motivated to suppress self-generated noise. When listening for a $250-\mathrm{Hz}$ signal, the wrongright difference at $250 \mathrm{~Hz}$ tended to be greater than zero for all age groups, while differences at other frequencies were elevated only for 4- to 6-year-olds and 7- to 10-year-olds. There are at least two reasons why this might be the case: self-generated noise could reduce sensitivity by energetically masking the signal, or increased self-generated noise and reduced sensitivity could reflect a third factor, such as restless inattention.

Another finding consistent with an interpretation in terms of energetic masking is that the pattern of wrong-right differences appears to be tuned as a function of frequency at $250 \mathrm{~Hz}$, and to a lesser degree at $1000 \mathrm{~Hz}$. Although there is little evidence of tuning at $4000 \mathrm{~Hz}$, the wrong-right difference tended to be greater in the lower analysis bands for the youngest listeners. This could reflect relatively greater effects of masking at low signal frequencies and other factors at high signal frequencies. One caveat is that this argument assumes that masking arises predominantly from noise in the analysis band centered on the signal, but this assumption may not strictly hold for young children; there is some evidence that young children experience off-frequency masking to a greater extent than adults (Leibold and Neff 2011).

At all signal frequencies, there was a trend for the wrong-right difference to be lower in older listeners. These results are consistent with the idea that older listeners are more adept at suppressing low-frequency self-generated noise when listening for a sound near threshold. This interpretation receives additional support from the observation that the difference in low-frequency noise levels before and after the response interval are larger for older listeners. Variability in levels of self-generated noise within child listeners are small relative to differences in behavioral thresholds, however. For the youngest group of listeners, the wrong-right difference at $250 \mathrm{~Hz}$ is on the order of $6.7 \mathrm{~dB}$, whereas the adult-child threshold difference is $18.0 \mathrm{~dB}$. One implication of this finding is that strategies for reducing effects of 
self-generated noise may not be able to completely eliminate its effects. For the example of $250 \mathrm{~Hz}$, discarding responses on the $50 \%$ of trials with the highest levels of self-generated noise would not fully counteract the effects of greater noise in young children than adults.

\section{Conclusions}

The present results provide evidence that self-generated noise contributes to the protracted maturation of low-frequency tone detection in quiet. It remains to be seen whether higher levels of self-generated noise could play a role in other psychoacoustic phenomena associated with prolonged development at low frequencies, such as temporal integration (He et al. 2010), backward masking (Buss et al. 2013), and identification of vowels characterized by low-frequency formants (Sussman 2001).

One practical implication of the present results is the possibility that threshold elevation due to increased levels of self-generated noise could be mistaken for minimal or mild hearing loss, particularly at low frequencies. It is widely recognized that appropriate and timely treatment of hearing loss is crucial for positive outcomes in infants and children (Moeller $2000)$, and there is growing recognition that even minimal loss (20-40 dB HL) can have a lasting detrimental effect on children's language, psychosocial, and academic development (Bess et al. 1998; Lewis et al. 2015). It would be useful to develop strategies for differentiating sensorineural hearing loss, masking due to self-generated noise, and flagging attention. For example, measurement of sensorineural hearing loss may be improved by monitoring levels of self-generated noise during testing, and rejecting or reducing the weight given to responses associated with high noise levels. This is similar to strategies employed in assessing otoacoustic emissions (e.g., Keefe and Ling 1998). Consideration of patient factors, such as attention deficit disorder or labored breathing, could also be beneficial when interpreting elevated low-frequency thresholds.

Another practical implication of the present results concerns the choice of transducers when evaluating auditory sensitivity in children and adults. Occluding the ear tends to enhance the effects of self-generated noise, such that low-frequency thresholds are higher when measured with earphones than in the free-field; this effect, observed in adults, was formerly referred to as "the missing $6 \mathrm{~dB}$ " (Block et al. 2004; Killion 1978). Whereas the effect of transducer is on the order of $5 \mathrm{~dB}$ for adults (Block et al. 2004; Killion 1978; Tillman et al. 1966), is could be substantially larger in young children. This prediction is presently being evaluated.

\section{Acknowledgements}

This work was supported by NIH NIDCD Grant No. R01 DC014460 (EB). Thanks to Doug Keefe, Michael Gorga, Ryan McCreery, and three anonymous reviewers for providing helpful comments on this manuscript.

\section{References}

Abdala C, Folsom RC. The development of frequency resolution in humans as revealed by the auditory brain-stem response recorded with notched-noise masking. J Acoust Soc Am. 1995; 98:921-930. [PubMed: 7642831]

ANSI. ANSI S3.6-2010, American National Standard Specification for Audiometers. American National Standards Institute; New York: 2010. 
Bargones JY, Werner LA, Marean GC. Infant psychometric functions for detection: Mechanisms of immature sensitivity. J Acoust Soc Am. 1995; 98:99-111. [PubMed: 7608411]

Bell-Lehmkuhler B, Meinke DK, Sedey A, et al. Reliability of audiometric thresholds obtained with insert earphones when used by certified audiometric technicians. Noise Health. 2009; 11:59-68. [PubMed: 19265255]

Berger EH, Kerivan JE. Influence of physiological noise and the occlusion effect on the measurement of real-ear attenuation at threshold. J Acoust Soc Am. 1983; 74:81-94. [PubMed: 6886201]

Bess FH, Dodd-Murphy J, Parker RA. Children with minimal sensorineural hearing loss: prevalence, educational performance, and functional status. Ear Hear. 1998; 19:339-354. [PubMed: 9796643]

Block MG, Killion MC, Tillman TW. The "missing 6 dB" of Tillman, Johnson, and Olsent was found -- 30 years ago. Seminars in Hearing. 2004; 25:7-16.

Bonino AY, Leibold LJ, Buss E. Release from perceptual masking for children and adults: Benefit of a carrier phrase. Ear Hear. 2013; 34:3-14. [PubMed: 22836239]

Buren M, Solem BS, Laukli E. Threshold of hearing $(0.125-20 \mathrm{kHz})$ in children and youngsters. Br J Audiol. 1992; 26:23-31. [PubMed: 1586846]

Buss E, He S, Grose JH, et al. The monaural temporal window based on masking period pattern data in school-aged children and adults. J Acoust Soc Am. 2013; 133:1586-1597. [PubMed: 23464028]

Elliott LL, Katz D. Children's pure-tone detection. J Acoust Soc Am. 1980; 67:343-344. [PubMed: 7354200]

Feigin JA, Kopun JG, Stelmachowicz PG, et al. Probe-tube microphone measures of ear-canal sound pressure levels in infants and children. Ear Hear. 1989; 10:254-258. [PubMed: 2776986]

Fior R. Physiological maturation of auditory function between 3 and 13 years of age. Audiol. 1972; 11:317-321.

Fletcher H. Auditory patterns. Review of Modern Physics. 1940; 12:47-65.

Fria TJ, Cantekin EI, Eichler JA. Hearing acuity of children with otitis media with effusion. Arch Otolaryngol. 1985; 111:10-16. [PubMed: 4038450]

Gavriely N, Palti Y, Alroy G. Spectral characteristics of normal breath sounds. J Appl Physiol Respir Environ Exerc Physiol. 1981; 50:307-314. [PubMed: 7204204]

Haapaniemi JJ. The hearing threshold levels of children at school age. Ear Hear. 1996; 17:469-477. [PubMed: 8979035]

Hall JW 3rd, Buss E, Grose JH. Informational masking release in children and adults. J Acoust Soc Am. 2005; 118:1605-1613. [PubMed: 16247871]

Handžić J, Radić B, Bagatin T, et al. Hearing in children with otitis media with effusion--clinical retrospective study. Coll Antropol. 2012; 36:1273-1277. [PubMed: 23390821]

He S, Buss E, Hall JW. Monaural temporal integration and temporally selective listening in children and adults. J Acoust Soc Am. 2010; 127:3643-3653. [PubMed: 20550263]

Keefe DH, Ling R. Double-evoked otoacoustic emissions. II. Intermittent noise rejection, calibration and ear-canal measurements. J Acoust Soc Am. 1998; 103:3499-3508. [PubMed: 9637034]

Killion MC. Revised estimate of minimum audible pressure: Where is the "missing $6 \mathrm{~dB}$ "? J Acoust Soc Am. 1978; 63:1501-1508. [PubMed: 690329]

Leibold LJ, Neff DL. Masking by a remote-frequency noise band in children and adults. Ear Hear. 2011; 32:663-666. [PubMed: 21336137]

Lewis DE, Stelmachowicz PG. Real ear to 6-cm3 coupler differences in young children. J Speech Hear Res. 1993; 36:204-209. [PubMed: 8450659]

Lewis DE, Valente DL, Spalding JL. Effect of minimal/mild hearing loss on children's speech understanding in a simulated classroom. Ear Hear. 2015; 36:136-144. [PubMed: 25170780]

Loeb M, Dickson C. Factors influencing the practice effect for auditory thresholds. J Acoust Soc Am. 1961; 33:917-921.

Lonsbury-Martin BL, Martin GK, McCoy MJ, et al. Otoacoustic emissions testing in young children: Middle-ear influences. Am J Otol. 1994; 15:13-20.

Maxon AB, Hochberg I. Development of psychoacoustic behavior: Sensitivity and discrimination. Ear Hear. 1982; 3:301-308. [PubMed: 7152153] 
Mayer DL, Dobson V. Visual acuity development in infants and young children, as assessed by operant preferential looking. Vision Res. 1982; 22:1141-1151. [PubMed: 7147725]

Moeller MP. Early intervention and language development in children who are deaf and hard of hearing. Pediatrics. 2000; 106:E43. [PubMed: 10969127]

Moller AR, Rollins PR. The non-classical auditory pathways are involved in hearing in children but not in adults. Neurosci Lett. 2002; 319:41-44. [PubMed: 11814649]

Moore JK, Linthicum FH Jr. The human auditory system: A timeline of development. Int J Audiol. 2007; 46:460-478. [PubMed: 17828663]

Moulin LK. The effects of physiological noise on the auditory threshold. J Speech Hear Res. 1972; 15:837-844. [PubMed: 4652406]

Müller R, Fleischer G, Schneider J. Pure-tone auditory threshold in school children. Eur Arch Otorhinolaryngol. 2012; 269:93-100. [PubMed: 21604074]

Norton SJ, Widen JE. Evoked otoacoustic emissions in normal-hearing infants and children: emerging data and issues. Ear Hear. 1990; 11:121-127. [PubMed: 2340965]

Nozza RJ, Wilson WR. Masked and unmasked pure-tone thresholds of infants and adults: Development of auditory frequency selectivity and sensitivity. J Speech Hear Res. 1984; 27:613622. [PubMed: 6521469]

Oh EL, Wightman F, Lutfi RA. Children's detection of pure-tone signals with random multitone maskers. J Acoust Soc Am. 2001; 109:2888-2895. [PubMed: 11425131]

Okabe K, Tanaka S, Hamada H, et al. Acoustic impedance measurement on normal ears of children. J Acoust Soc Jap. 1988; 9:287-294.

Pujol R, Lavigne-Rebillard M, Uziel A. Development of the human cochlea. Acta Oto-laryngol. 1991; 482(Supp):7-12. discussion 13.

Ren T, Zhang M, Nuttall AL, et al. Heart beat modulation of spontaneous otoacoustic emissions in guinea pig. Acta Otolaryngol. 1995; 115:725-731. [PubMed: 8749191]

Schneider BA, Trehub SE, Morrongiello BA, et al. Auditory sensitivity in preschool children. J Acoust Soc Am. 1986; 79:447-452. [PubMed: 3950197]

Schneider BA, Trehub SE, Morrongiello BA, et al. Developmental changes in masked thresholds. J Acoust Soc Am. 1989; 86:1733-1742. [PubMed: 2808922]

Shaw, EAG. Handbook of Sensory Physiology. Springer-Verlag; Berlin: 1974. The external ear; p. 455-490.

Stekelenburg JJ, van Boxtel A. Inhibition of pericranial muscle activity, respiration, and heart rate enhances auditory sensitivity. Psychophysiol. 2001; 38:629-641.

Sussman JE. Vowel perception by adults and children with normal language and specific language impairment: based on steady states or transitions? J Acoust Soc Am. 2001; 109:1173-1180. [PubMed: 11303931]

Tillman TW, Johnson RM, Olsen WO. Earphone versus sound-field threshold sound-pressure levels for spondee words. J Acoust Soc Am. 1966; 39:125-133. [PubMed: 5904527]

Trehub SE, Schneider BA, Morrongiello BA, et al. Auditory sensitivity in school-age children. J Exp Child Psychol. 1988; 46:273-285. [PubMed: 3171476]

Trehub SE, Schneider BA, Morrongiello BA, et al. Developmental changes in high-frequency sensitivity. Audiol. 1989; 28:241-249.

Voss SE, Herrmann BS. How does the sound pressure generated by circumaural, supra-aural, and insert earphones differ for adult and infant ears? Ear Hear. 2005; 26:636-650. [PubMed: 16377999]

Walsh KP, Pasanen EG, McFadden D. Selective attention reduces physiological noise in the external ear canals of humans. I: auditory attention. Hear Res. 2014; 312:143-159. [PubMed: 24732069]

Watson CS, Franks JR, Hood DC. Detection of tones in the absence of external masking noise. I. Effects of signal intensity and signal frequency. J Acoust Soc Am. 1972; 52:633-643.

Wightman FL, Kistler DJ, O'Bryan A. Individual differences and age effects in a dichotic informational masking paradigm. J Acoust Soc Am. 2010; 128:270-279. [PubMed: 20649222]

Ear Hear. Author manuscript; available in PMC 2017 November 01. 


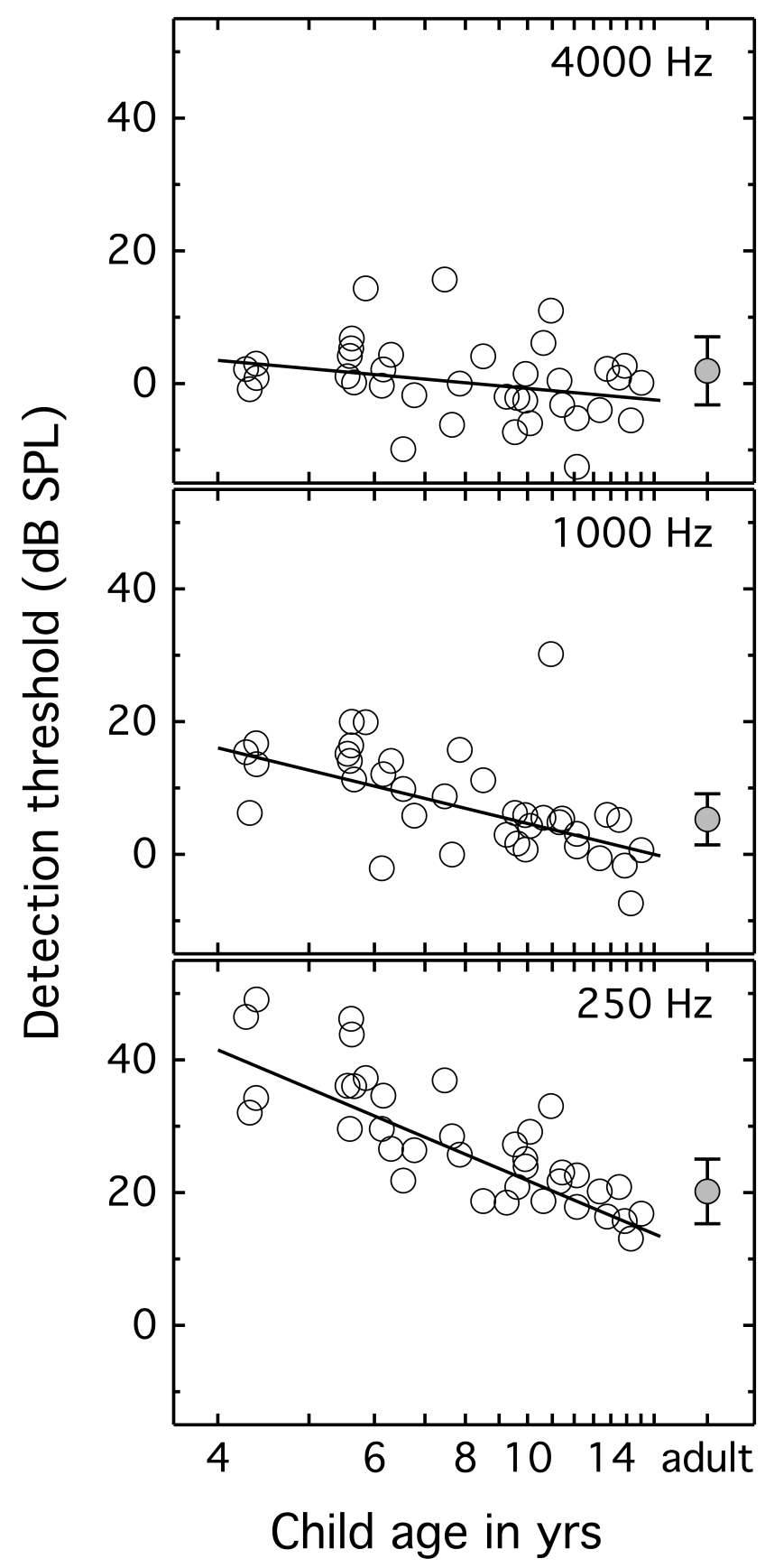

Figure 1.

Open circles show mean detection thresholds for individual child listeners, plotted as a function of age on a log scale. Lines are fitted to threshold as a function of child age on a log scale. Filled circles at the far right show the group mean for adult listeners, and error bars indicate plus and minus one standard deviation. Results for the three signal frequencies are shown in separate panels. 


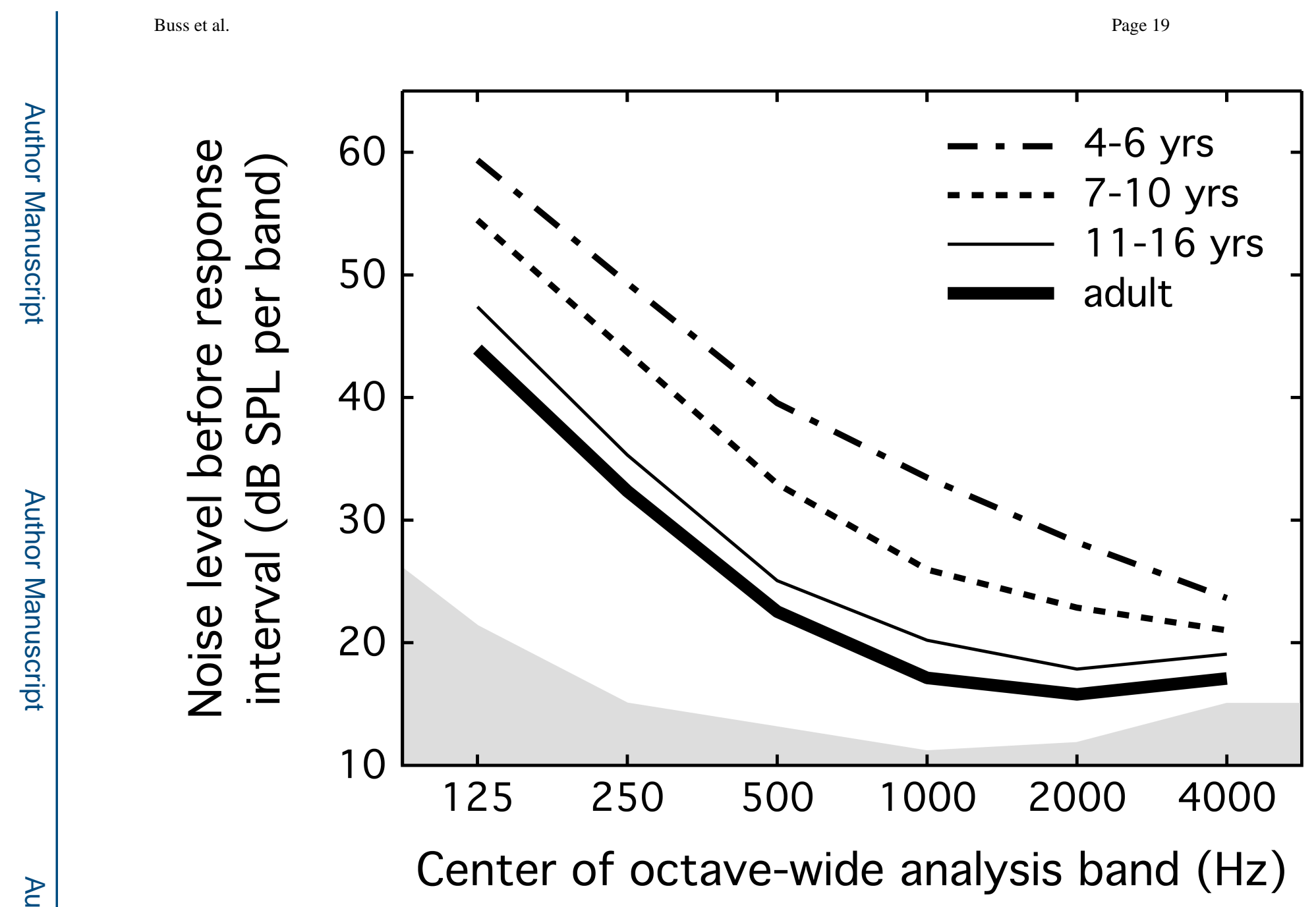

Figure 2.

The mean level of self-generated noise recorded prior to the presentation of the signal is plotted for neighboring octave-wide bands as a function of center frequency. Shading indicates the noise floor, and lines indicate results for the four age groups, as defined in the legend. 


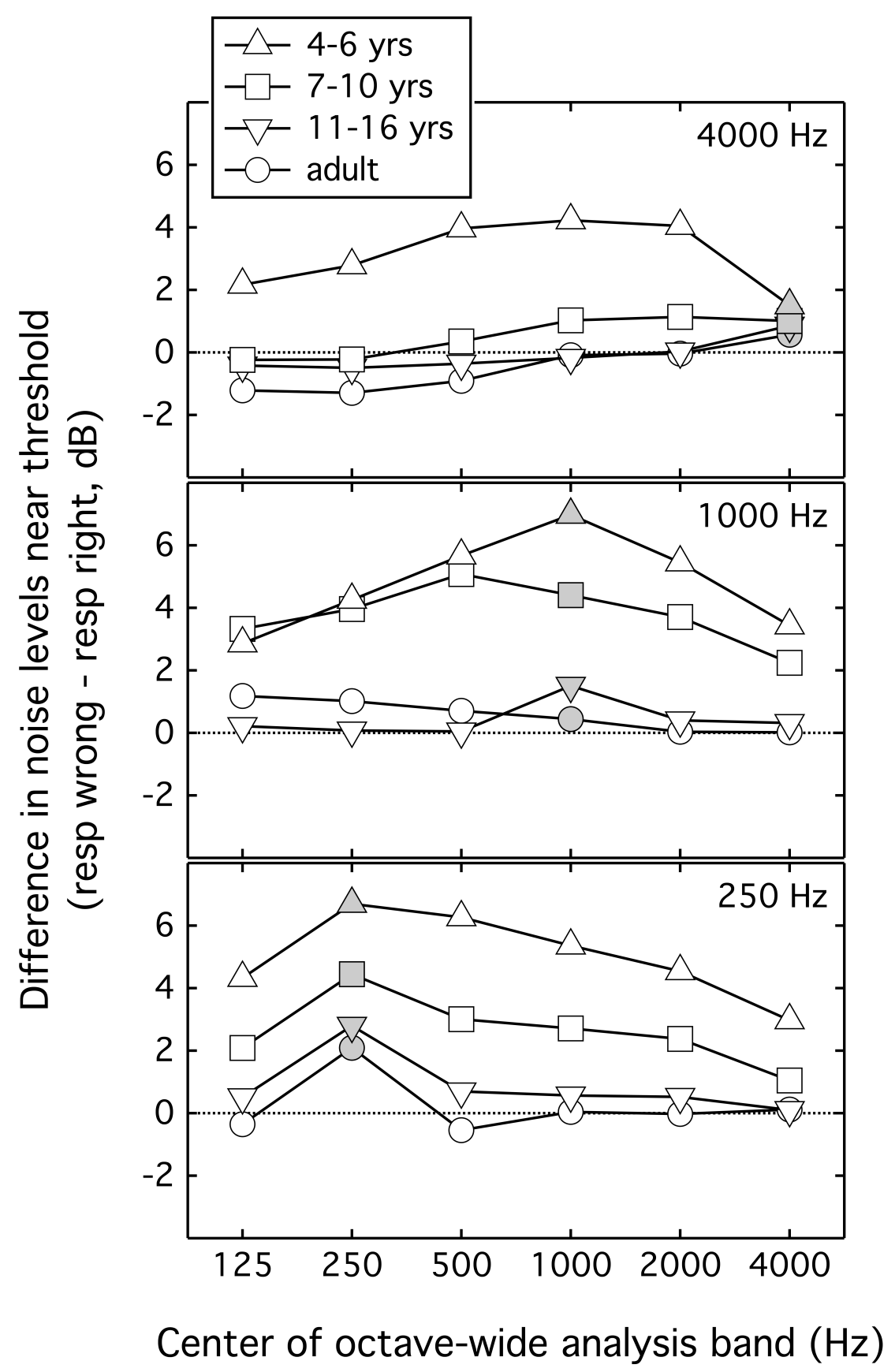

Figure 3.

The difference in mean levels of self-generated noise according to listener response -- wrong minus right -- is plotted as a function of the center frequency of the octave-wide analysis band. This analysis excluded trials before the second track reversal and omitted the octavewide band centered on the signal frequency. Symbols reflect the listener age group, as defined in the legend, and data associated with each of three signal frequencies are shown in separate panels. 


\section{Table 1}

Levels of self-generated noise in octave-wide bands for intervals before the interval identified by the listener as the signal interval. The mean values and standard deviations are reported in AB SPL for each age group and signal frequency.

\begin{tabular}{lrrrrrr} 
& \multicolumn{6}{c}{ Frequency $(\mathbf{H z})$} \\
\cline { 2 - 7 } Age group & $\mathbf{1 2 5}$ & $\mathbf{2 5 0}$ & $\mathbf{5 0 0}$ & $\mathbf{1 0 0 0}$ & $\mathbf{2 0 0 0}$ & $\mathbf{4 0 0 0}$ \\
\hline 4-6 yrs & 59.4 & 49.3 & 39.5 & 33.5 & 28.2 & 23.6 \\
& 5.4 & 6.4 & 7.0 & 6.2 & 4.8 & 3.0 \\
\hline $7-10$ yrs & 54.5 & 43.7 & 33.0 & 26.0 & 22.9 & 21.0 \\
& 4.3 & 4.6 & 5.6 & 4.9 & 3.7 & 2.5 \\
\hline $11-16$ yrs & 47.4 & 35.3 & 25.1 & 20.2 & 17.9 & 19.1 \\
& 4.0 & 5.3 & 4.9 & 3.5 & 2.0 & 1.6 \\
\hline adults & 44.0 & 32.4 & 22.6 & 17.1 & 15.8 & 17.1 \\
& 3.9 & 5.0 & 4.4 & 2.1 & 1.6 & 0.8 \\
\hline
\end{tabular}

Ear Hear. Author manuscript; available in PMC 2017 November 01. 


\section{Table 2}

Levels of self-generated noise in octave-wide bands were compared for intervals before and after the interval identified by the listener as the signal interval. The mean difference (after-before) is reported below in $\mathrm{dB}$ for each age group and signal frequency. Larger numbers reflect higher noise levels after the interval identified by the listener as containing the signal.

\begin{tabular}{lcccccc} 
& \multicolumn{6}{c}{ Frequency $(\mathbf{H z})$} \\
\cline { 2 - 7 } Age group & $\mathbf{1 2 5}$ & $\mathbf{2 5 0}$ & $\mathbf{5 0 0}$ & $\mathbf{1 0 0 0}$ & $\mathbf{2 0 0 0}$ & $\mathbf{4 0 0 0}$ \\
\hline 4-6 yrs & 3.2 & 4.2 & 4.1 & 3.1 & 2.5 & 1.7 \\
\hline $7-10$ yrs & 3.8 & 4.1 & 3.8 & 3.0 & 1.7 & 0.7 \\
\hline $11-16$ yrs & 5.5 & 5.9 & 4.8 & 3.3 & 1.4 & 0.4 \\
\hline adults & 8.1 & 9.2 & 8.1 & 5.8 & 3.6 & 1.6 \\
\hline
\end{tabular}

Ear Hear. Author manuscript; available in PMC 2017 November 01. 